\title{
Application of the Bayesian Model Averaging in Analyzing Freeway Traffic Incident Clearance Time for Emergency Management
}

\author{
Yajie Zou, ${ }^{1}$ Bo Lin $\left(\mathbb{D},{ }^{1}\right.$ Xiaoxue Yang, ${ }^{1}$ Lingtao $W u,{ }^{2}$ Malik Muneeb Abid, ${ }^{3}$ and Jinjun Tang $\mathbb{}{ }^{4}$ \\ ${ }^{1}$ Key Laboratory of Road and Traffic Engineering of Ministry of Education, Tongji University, Shanghai 201804, China \\ ${ }^{2}$ Texas A\&M Transportation Institute, Texas A\&M University System, 3135, TAMU, College Station, TX 77843-3135, USA \\ ${ }^{3}$ Department of Civil Engineering, College of Engineering and Technology, University of Sargodha, Sargodha, Pakistan \\ ${ }^{4}$ The School of Traffic \& Transportation Engineering, Central South University, Changsha 410075, China \\ Correspondence should be addressed to Jinjun Tang; jinjuntang@csu.edu.cn
}

Received 11 December 2020; Revised 31 January 2021; Accepted 1 March 2021; Published 11 March 2021

Academic Editor: Yong Wang

Copyright (C) 2021 Yajie Zou et al. This is an open access article distributed under the Creative Commons Attribution License, which permits unrestricted use, distribution, and reproduction in any medium, provided the original work is properly cited.

\begin{abstract}
Identifying the influential factors in incident duration is important for traffic management agency to mitigate the impact of traffic incidents on freeway operation. Previous studies have proposed a variety of approaches to determine the significant factors for traffic incident clearance time. These methods commonly select a single "true" model among a majority of alternative models based on some model selection criteria. However, the conventional methods generally neglect the uncertainty related to the choice of models. This paper proposes a Bayesian Model Averaging (BMA) model to account for model uncertainty by averaging all plausible models using posterior probability as the weight. The BMA model is used to analyze the 2,584 freeway incident records obtained from I-5 corridor in Seattle, WA, USA. The results show that the BMA approach has the capability of interpreting the causal relationship between explanatory variables and clearance time. In addition, the BMA approach can provide better prediction performance than the Cox proportional hazards model and the accelerated failure time models. Overall, the findings in this study can be useful for traffic emergency management agency to apply an alternative methodology for predicting traffic incident clearance time when model uncertainty is considered.
\end{abstract}

\section{Introduction}

Improving the efficiency of the traffic incident management is a common measure to alleviate traffic congestion [1-3]. Although the definition and components of traffic incident duration vary in different studies, clearance time is regarded as the most uncontrollable component [4]. As a phase of incident duration, it depends on the unique factors of each individual incident [5]. Thus, understanding the effects of influential factors and accurate incident clearance time prediction are essential to evaluate traffic incident management strategy [6].

A variety of approaches have been utilized to predict incident clearance time and analyze the effects of influential factors during the past several decades. In general, these approaches can be classified into statistical methods [7] and machine learning methods $[8,9]$. Statistical methods generally can explain the mechanism between independent and dependent variables based on the rigorous mathematical formula $[10,11]$. From a methodological perspective, regression analysis and hazard-based methods are the two main statistical methods to analyze incident duration data. Regression methods were widely used for incident duration prediction in previous studies, such as linear regression $[12,13]$. To overcome the simple linear assumption between incident clearance time and explanatory variables, researchers proposed the hazard-based duration models (HBDM) to predict the incident duration precisely and explore the influence of significant factors on the incident duration, such as the Cox Proportional Hazards (PH) model and the Accelerated Failure Time (AFT) model [14]. Nam and Mannering [5] applied different distributions in HBDMs to analyze incident duration based on 681 incidents from Washington State's incident response team program. 
Lee and Fazio [15] used a proportional hazard-based Coxregression model to analyze the effect of explanatory variables on response time and clearance time, respectively. Li and Shang [16] selected the best-fit model from 17 candidate different AFT models with different distributions according to the Bayesian Information Criterion (BIC) values, to investigate the effective factors of each incident and predict the time of each incident phase. Ghosh et al. [17] pointed out that the AFT model with a generalized F distribution outperformed other five parametric hazard-based duration models when analyzing the incident clearance time data collected in Michigan. Haule et al. [18] investigated the effects of significant factors on incident clearance time and selected the best-fit model from three AFT models with different distributions in terms of the Akaike information criterion (AIC).

Recently, large quantities of alternative methods have been introduced under the framework of the HBDMs in recent years. For example, a 2-component log-logistic finite mixture model was used by Zou et al. [19] to analyze the incident clearance time data obtained from the Washington Incident Tracking System (WITS), and the model could be better in accounting for the heterogeneities compared to the standard survival model. Different from conventional conditional-mean HBDMs, the quantile regression $(\mathrm{QR})$ model can estimate the changing influence of explanatory variables on incident clearance time as each quantile of the incident duration distribution varied $[20,21]$. And a copulabased approach was proposed to accommodate the correlation between incident clearance and other phases of incident duration, and the results showed that the copula models outperformed the log-normal AFT model in predicting the clearance time $[22,23]$.

Compared with the statistical methods, machine learning approaches can be employed to overcome complex nonlinear relationships between incident clearance time and explanatory variables without prior assumptions about input data $[24,25]$. In the previous studies, K-Nearest Neighbor algorithm (KNN) and Bayesian networks were used to predict the incident clearance time [26-28]. Besides, decision trees (DT) models can specify the relative importance of different explanatory variables associated with response variables and are widely used to analyze the traffic incident duration [29]. Nevertheless, the structure of the decision tree highly depends on the data, resulting in instability. To overcome the imbalanced traffic incident duration data problem of the single-tree-based method, Ma et al. [30] found that the gradient boosting decision tree model has a superior performance in model interpretation and prediction accuracy to conventional DT models [31]. Also based on traditional DT models, the extreme gradient boosting machine algorithm was applied to analyze and predict the clearance time data [32, 33]. However, the machine learning models are usually not capable of interpreting the mechanism between estimator and explanatory variables.

Overall, the hazard-based models and the tree-based models are two commonly used statistical and machine learning models in traffic accident duration analysis. However, these aforementioned approaches generally failed to take account of the model uncertainty (e.g., the AFT models with Gamma, Weibull, or log-logistic model) [34]. In other words, traditional methods typically assumed that the model to be estimated is the "true" model and then made tests among a majority of alternative "true" model according to some criteria like BIC [35]. Consequently, it is important to consider the uncertainty between candidate models, especially when these model are considered reasonable in spite of difference in predictions [36]. Otherwise, the resulting model estimates may be biased and lead to erroneous inference in the analysis of incident clearance time. The Bayesian Model Averaging (BMA) proposed by Draper [37] provided a statistical theoretical basis for solving the model uncertainty problem in econometric modeling. This approach combines and averages all plausible models considered (models with various combinations of influential variables) through setting different prior probability distributions [38] and has been widely used in various fields, such as water main failures prediction [39], firm default prediction [40], and chemical engineering [41].

The objective of this research is to apply the BMA method to account for both model and parameter uncertainty when modeling the incident clearance time. To examine the proposed approach, 2,584 freeway incident records obtained from I-5 corridor in Seattle are analyzed. Estimation and prediction results from the proposed BMA model and conventional HBDMs are then compared and analyzed.

\section{Methodology}

This section describes the general features of the BMA, two conventional HBDMs and Occam's Window Method. The last method is one of the sampling techniques used in the model space.

2.1. Bayesian Model Averaging. BMA uses posterior probability as the weight to average all plausible models considered. Thus, let $M=\left\{M_{1}, \ldots, M_{K}\right\}$ denote the set of all models and let $y$ denote the future observed value of the incident clearance time using new input data. Then, in accordance with the law of total probability, the probability density function (PDF) of $y$ under the observed dataset $D$ is

$$
p(y \mid D)=\sum_{k=1}^{K} p\left(y \mid M_{k}, D\right) p\left(M_{k} \mid D\right),
$$

where $p\left(y \mid M_{k}, D\right)$ is the mean of the posterior distribution of $y$ based on candidate model $M_{k}$, which is the output of BMA approach. And $p\left(M_{k} \mid D\right)$ is the probability of the correct prediction model $M_{k}$, which is also referred to as the posterior model probability (PMP). And in model space $M$, $\sum_{k=1}^{K} p\left(M_{k} \mid D\right)=1$. The PMP is given by Bayes' rule:

$$
p\left(M_{k} \mid D\right)=\frac{p\left(M_{k}\right) p\left(D \mid M_{k}\right)}{\sum_{l=1}^{K} p\left(M_{l}\right) p\left(D \mid M_{l}\right)},
$$

where 


$$
p\left(D \mid M_{k}\right)=\int p\left(D \mid \theta_{k}, M_{k}\right) p\left(\theta_{k} \mid M_{k}\right) \mathrm{d} \theta_{k},
$$

is the marginal model likelihood for the given model $M_{k}$ and $\theta_{k}$ is the vector of parameters of the model $M_{k}$. Additionally, $p\left(\theta_{k} \mid M_{k}\right)$ and $p\left(D \mid \theta_{k}, M_{k}\right)$ are the prior density of $\theta_{k}$ and the likelihood given the model $M_{k}$, respectively. $p\left(M_{k}\right)$ refers to the prior probability that $M_{k}$ is considered as the "true" model. The posterior mean and variance of $y$ are presented as follows:

$$
\begin{aligned}
E[y \mid D] & =\sum_{k=1}^{K} E\left(y \mid D, M_{k}\right) p\left(M_{k} \mid D\right), \\
\operatorname{Var}[y \mid D] & =\sum_{k=1}^{K}\left(\operatorname{Var}\left[y \mid D, M_{k}\right]+E\left[y \mid D, M_{k}\right]^{2}\right) p\left(M_{k} \mid D\right)-E[y \mid D]^{2} .
\end{aligned}
$$

Based on the previous work, the size of model space makes the summation of (1) impractical. In order to solve this problem, Occam's window method is used to select an appropriate collection of candidate models from the model space and will be introduced in the following section.

2.2. Occam's Window Method. Occam's window approach was proposed by Madigan and Raftery [42] to reduce the number of models with low posterior probability in the model space $M$. Two basic principles were conducted to eliminate models that predict far less well than their competitors.

First, if the PMP for the model $M_{k}$ in the model space $M$ is calculated far lower than the model that provides the highest PMP, model $M_{k}$ is discarded from the model space $M$. Those models failing to satisfying the formula

$$
A^{\prime}=\left\{M_{k}: \frac{\max _{l}\left\{p\left(M_{l} \mid D\right)\right\}}{p\left(M_{k} \mid D\right)} \leq C\right\}
$$

should be excluded from equation (1). The $\max _{l}\left\{p\left(M_{l} \mid D\right)\right\}$ refers to the model with the highest PMP, and the value of $C$ equals 20 since it is determined by the data analyst.

Second, if the PMPs of complex models are lower than their simpler submodels using Occam's razor method, these models should be discarded from model space $M$ belonging to

$$
B=\left\{M_{k}: \exists M_{l} \in M, M_{l} \subset M_{k}, \frac{p\left(M_{l} \mid D\right)}{p\left(M_{k} \mid D\right)}>1\right\} .
$$

Therefore, formula (1) can be expressed as

$$
p(y \mid D)=\sum_{M_{k} \in A} p\left(y \mid M_{k}, D\right) p\left(M_{k} \mid D\right),
$$

where $A=A \prime \backslash B \in M$.

Additionally, the leaps and bounds algorithm is applied to implement the above principles as the search strategy. Interested readers can see the companion paper for more details about this algorithm [43].
2.3. Cox Proportional Hazards (PH) Model. The Cox proportional hazard $(\mathrm{PH})$ model is one of the most commonly used semiparametric survival analysis models. This model is used for investigating the relationship between survival time of respondents and predictor variables [44] and is given as follows:

$$
h(t, X)=h_{0}(t) \exp \left(\sum_{i=1}^{p} \beta_{i} X_{i}\right),
$$

where $t$ is the survival time, $X=\left(X_{1}, X_{2}, \ldots, X_{P}\right)$ represents a vector of explanatory variables, $\beta$ are the coefficients to be estimated, which measure the impact of the p-covariates, $h(t, X)$ is the hazard function, $h_{0}(t)$ is an unknown baseline hazard, that is, the value of the hazard if all the $X_{i}$ are equal to zero, and equation (8) can also be written as

$$
\log \left(\frac{h(t, X)}{h_{0}(t)}\right)=\sum_{i=1}^{p} \beta_{i} X_{i}
$$

where $\left(h(t, X) / h_{0}(t)\right)$ is defined as hazard ratios (HR), in which any two individuals are constant over time according to the assumption of the Cox PH model.

2.4. Accelerated Failure Time (AFT) Model. Based on the hazard function, the AFT model assumes that the log of the survival times is affected linearly by the covariates of $X$ and can be clearly written as

$$
\ln (t)=\xi_{i} X_{i}+\varepsilon,
$$

where $\xi$ are the coefficients to be estimated, and $\varepsilon$ is an error term. There are kinds of different parametric distributions (e.g., Weibull, log-normal, and log-logistic models), which can be used for the AFT model.

For comparison purposes, the incident data were also analyzed using the Cox PH model and the log-logistic AFT model. In the previous study, it is suggested that the loglogistic AFT model outperformed the other two AFT models (with Weibull or log-normal distribution) in terms of the goodness-of-fit statistics and predictive performance. Therefore, the two conventional HBDMs were finally selected as the benchmark models in this study.

2.5. Data Description. In this study, the incident data obtained from the I-5 corridor between Boeing Access Road and the Seattle Central Business District were retrieved from the WITS, which is used to manage the incident log data. The reason for choosing this site is the heavy traffic demand and frequent incident-induced traffic congestion events. Additionally, in previous studies, Tang et al. [32] used the data source to analyze the influence of explanatory variables and examine the prediction performance of the proposed model. And Hou et al. [45] analyzed the time-varying effects of significant variables based on this dataset. A total of 2,584 valid incidents from 1 January to 31 December 2009 were selected from the WITS dataset including 15 categorical 
candidate explanatory variables. Additionally, in this dataset, the mean and median of the incident clearance time were 13.58 minutes and 9 minutes. The minimum and maximum values were 1 minutes and 382 minutes, respectively. And the standard deviation was 17.35 minutes. The key information of candidate variables is presented in Table 1 .

\section{Results and Discussion}

This section describes the modeling results for the BMA approach, Cox PH model, and the log-logistic AFT model. In terms of the model averaging strategies, it is assumed that all possible combinations of 15 candidate explanatory variables are equally likely a priori. As previously mentioned, Occam's window method is implemented to exclude complex models that perform far less well than their simpler competitors and discard them from the model space. The results show that the BMA approach and two benchmark models differ in interpreting the explanatory variables based on the same dataset, and the former can provide better prediction performance. All statistical analyses were conducted using statistical software R.

Table 2 lists the posterior means, standard deviations, and posterior effect probabilities $P(\beta \neq 0 \mid D)$ for the coefficients related to different explanatory variables by using the proposed method. The posterior effect probability $P(\beta \neq 0 \mid D)$ equals the sum of the posterior probabilities for all the selected models containing that explanatory variable. For example, the posterior effect probabilities associated with the explanatory variable single lane blocked have $7.8 \%$ of its mass at zero. Unlike other statistical models, it can be observed that the variable "single lane blocked" is not included in all the selected models, although it has a significant impact on incident clearance time. 20 different models with the highest PMP were finally selected by Occam's window approach, among which five optimal models (PMP > 0.05) shown in Table 2 account for $59.69 \%$ of the total posterior probability. Additionally, the estimated coefficients for some variables (i.e., advised WSP, disabled vehicle, debris, heavy truck, weekends, winter, weather, and incident on HOV lane) are not listed in Table 2 because the $p$ values are larger than the significance level (0.05). It is found that model 1 with the largest PMP can account for $22.8 \%$ of the total posterior probability, which means that there exists quite a bit model uncertainty.

As shown, the model averaging results of the predictors for incident clearance time generate interesting interpretations. The posterior effect probabilities of seven explanatory variables equal $100 \%$. This result demonstrates that, for response time, traffic control, collision, multiple lane blocked, total closure, injury involved, and summer, all the selected models with the highest PMP contain these explanatory variables, and thus they are the main factors affecting the duration of traffic incident clearance. The positive coefficient in the results of the proposed model indicates that the hazard is higher, and the clearance time decreases with higher values of that variable.
It is clear that summer results in shorter clearance time, which means that the incident clearance time varies with the month of year and gets shorter in summer than other seasons, and for the remaining variables, they all result in longer clearance time.

As the BMA results in Table 2 indicate, we can see that response time shows a weak effect on incident clearance time, the coefficient of which implies that more time is required to prepare for incident response in congested traffic periods. The estimated coefficient -0.527 of traffic control on clearance time deserves attention to improve the measures undertaken by traffic incident management like incident response team (IRT) on directing upstream traffic around the incident.

Among the considered incident types, collision is the only one significant variable affecting the clearance time. And the involvement of fire and injury shows positive effect on incident clearance time. These results demonstrate that both fire and collision involvement can result in longer clearance time or smaller hazard rates and are generally the main factors causing traffic congestion.

Four types of lane closure except all travel lanes blocked all have a significant impact on incident clearance time. This is caused by the increasing operational complexity of the recovery process around the incident. On closer inspection, of all significant variables, total closure has the strongest impact on incident clearance time, which indicates that longer clearance time is generally associated with total closed lanes. To further explain the BMA model, the estimated survival probabilities are plotted in Figure 1.

Meanwhile, Tables 3 and 4 list parameter estimation results of the Cox PH model and the log-logistic AFT model for comparison purposes, respectively. The estimated coefficients for some variables are not listed in Tables 3 and 4 because the $p$ values are larger than the significance level (0.05). It is noted that, in the Cox model, the sign of the regression coefficient (coef) is opposite to that of the AFT model. The positive coefficient in the results of the Cox PH model indicates that the hazard is higher, and thus the clearance time decreases with higher values of that variable. Note that, different from the results of the BMA, all types of incident are significant in terms of $p$ values in the Cox PH model. Moreover, three lane closure types (single lane blocked, multiple lane blocked, and total closure), response time, traffic control, injury involved, fire involved, and work zone involved all result in longer clearance time or smaller hazard rates. Debris, abandoned vehicle, night, and summer all result in shorter incident clearance time or larger hazard rates. These findings are consistence with the previous study using quantile regression.

In the log-logistic AFT model, night is found statistically significant compared with the BMA model. And disabled vehicle, debris, and abandoned vehicle all result in longer clearance time in both survival models, while these variables are not statistically significant in the BMA model. The reason is that when model uncertainty is considered, their posterior effect probabilities from BMA are significantly lower than 
TABLe 1: The key information for valid incidents affected by candidate explanatory variables.

\begin{tabular}{|c|c|c|}
\hline Category & Variable & Value set \\
\hline \multirow{7}{*}{ Incident } & Incident type & (Disabled vehicle, debris, abandoned vehicle, collision and others ${ }^{\mathrm{a}}(=0)$ ) \\
\hline & Lane closure type & $\begin{array}{c}\text { (Single lane blocked, multiple lanes blocked, all travel lanes blocked and total } \\
\text { closure) }\end{array}$ \\
\hline & Injury involved & $(0,1)$ \\
\hline & Fire involved & $(0,1)$ \\
\hline & Work zone involved & $(0,1)$ \\
\hline & Heavy truck involved & $(0,1)$ \\
\hline & Time of the day & (Daytime $(=0)$, night $(22: 00-6: 00)(=1))$ \\
\hline \multirow{2}{*}{ Temporal } & Day of the week & (Weekday $(=0)$, weekend (Sat, Sun) $(=1)$ ) \\
\hline & Month of the year & (Summer (Jun, Jul, Aug), winter (Dec, Jan, Feb) and other seasons $(=0)$ ) \\
\hline Geographic & Incident on Hov lane $\mathrm{b}^{\mathrm{b}}$ & $(0,1)$ \\
\hline Environmental & Weather & (Rainy, snowy and other type $(=0)$ ) \\
\hline \multirow{3}{*}{ Traffic } & Peak hours $(6: 00-9: 00,15: 00-18: 00)$ & $(0,1)$ \\
\hline & Response time & $\mathrm{R}+$ \\
\hline & Traffic control & $(0,1)$ \\
\hline Operational & $\begin{array}{l}\text { Washington state patrol (WSP) } \\
\text { involved }\end{array}$ & $(0,1)$ \\
\hline
\end{tabular}

${ }^{\mathrm{a} O t h e r s ~ r e f e r ~ t o ~ c a r ~ f i r e s, ~ p o l i c e ~ a c t i v i t y, ~ a n d ~ m e d i c a l ~ e m e r g e n c y ~ o n ~ t h e ~ r o a d, ~ e t c . ~}{ }^{\mathrm{b}} \mathrm{Hov}$ lane refers to the vehicle lane with high-occupancy.

TABLE 2: Selected models with the highest posterior probabilities using BMA.

\begin{tabular}{|c|c|c|c|c|c|c|c|c|}
\hline Variable & $P(\beta \neq 0 \mid D)$ & $\mathrm{EV}^{\mathrm{a}}$ & $\mathrm{SD}^{\mathrm{b}}$ & Model1 & Model2 & Model3 & Model4 & Model5 \\
\hline Response time & 100.0 & -0.092 & 0.004 & -0.091 & -0.094 & -0.091 & -0.092 & -0.092 \\
\hline Traffic control & 100.0 & -0.527 & 0.062 & -0.536 & -0.509 & -0.533 & -0.502 & -0.533 \\
\hline Collision & 100.0 & -0.737 & 0.211 & -0.866 & -0.780 & -0.877 & -0.380 & -0.868 \\
\hline Single lane blocked & 92.2 & -0.182 & 0.078 & -0.173 & -0.217 & -0.176 & -0.216 & -0.182 \\
\hline Multiple lane blocked & 100.0 & -0.619 & 0.111 & -0.608 & -0.670 & -0.586 & -0.639 & -0.623 \\
\hline Total closure & 100.0 & -1.491 & 0.357 & -1.501 & -1.531 & -1.536 & -1.444 & -1.523 \\
\hline Injury involved & 100.0 & -0.485 & 0.123 & -0.486 & -0.484 & -0.475 & -0.500 & -0.484 \\
\hline Fire involved & 92.6 & -1.061 & 0.455 & -1.248 & -1.177 & -1.143 & -8.969 & -1.257 \\
\hline Summer & 100.0 & 0.170 & 0.045 & 0.176 & 0.171 & 0.173 & 0.163 & 0.173 \\
\hline$n \operatorname{Var}^{\mathrm{c}}$ & & & & 11 & 12 & 10 & 12 & 12 \\
\hline BIC & & & & -1488 & -1486 & -1486 & -1486 & -1485 \\
\hline PMP & & & & 0.228 & 0.117 & 0.106 & 0.086 & 0.060 \\
\hline
\end{tabular}

${ }^{a} \mathrm{EV}$ is posterior means. ${ }^{\mathrm{b}} \mathrm{SD}$ means the standard deviation. ${ }^{\mathrm{c}} \mathrm{nVar}$ refers to the number of variables in the $M_{k}$ model.

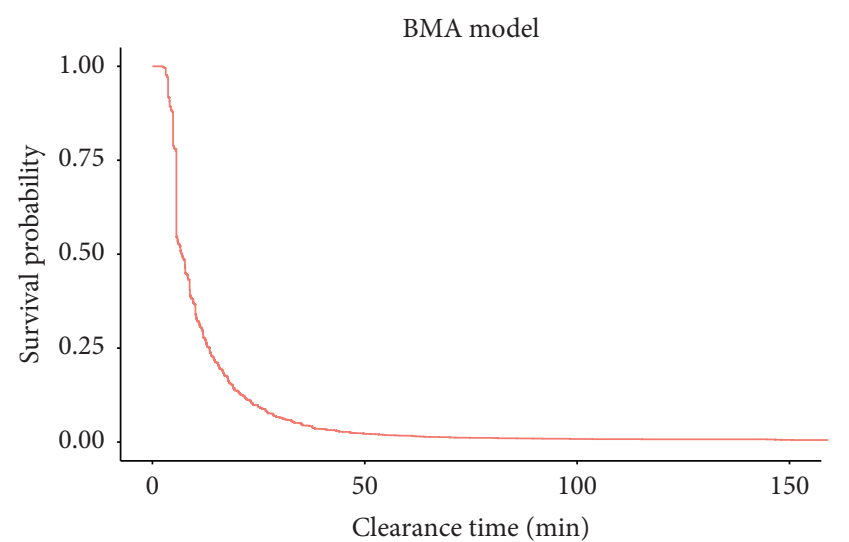

Figure 1: Estimated survival probability for the BMA model.

other variables. To further explain the Cox PH model, the log-logistic AFT model, the estimated survival probabilities are plotted in Figures 2 and 3.

The estimated coefficients from the Cox $\mathrm{PH}$, the loglogistic AFT, and BMA models can give macroscopic and valuable insights for incident duration time. For all travel lanes blocked, the estimated coefficient -0.796 of the Cox $\mathrm{PH}$ model and the coefficient 0.615 of the log-logistic AFT model indicate a strong impact on clearance time, while the posterior effect probability in the BMA model equals $8.2 \%$, which means that this variable is actually a less significant determinant of incident clearance time. Furthermore, the contributing factors resulting in longer clearance time can be identified, and thus traffic emergency management agency can improve their management planning and response strategies. As shown in Tables 2-4, the variables "total closure" and "fire involved" have a stronger positive impact on incident clearance time. Thus, when closing all lanes and fire involved traffic incidents are considered, more attention should be given to prevent further severe incidents.

More importantly, the posterior effect probability in the BMA model can overcome the overstatement of the evidence for an effect. The $p$ values for the coefficients indicate whether these relationships are statistically significant. In general, the $p$ values for the predictors for the single model are used as a measure of interpreting the effect on incident 
TABle 3: The estimated parameters for the Cox PH model ${ }^{\mathrm{a}}$.

\begin{tabular}{lcccc}
\hline \multirow{2}{*}{ Variable } & \multicolumn{4}{c}{ Cox PH model } \\
& Coef $^{\mathrm{b}}$ & $\mathrm{SE}$ & $z$-statistic & $p$ value \\
\hline Response time & -0.098 & 0.004 & -25.840 & $<2 e-16$ \\
Traffic control & -0.531 & 0.056 & -9.540 & $<2 e-16$ \\
Disabled vehicle & -0.268 & 0.115 & -2.340 & 0.019 \\
Debris & 0.465 & 0.132 & 3.520 & 0.000 \\
Abandoned vehicle & 0.272 & 0.128 & 2.130 & 0.034 \\
Collision & -0.636 & 0.125 & -5.070 & 0.000 \\
Single lane blocked & -0.291 & 0.060 & -4.890 & 0.000 \\
Multiple lane blocked & -0.736 & 0.103 & -7.120 & 0.000 \\
Total closure & -1.631 & 0.357 & -4.570 & 0.000 \\
Injury involved & -0.450 & 0.123 & -3.670 & 0.000 \\
Fire involved & -1.118 & 0.342 & -3.270 & 0.001 \\
Work zone involved & -0.459 & 0.184 & -2.490 & 0.013 \\
Night & 0.447 & 0.123 & 3.640 & 0.000 \\
Summer & 0.171 & 0.048 & 3.540 & 0.000 \\
\hline
\end{tabular}

${ }^{\mathrm{a}}$ Likelihood ratio test (LR): 1767 , and $p<2 e-16$. ${ }^{\mathrm{b}}$ Coef means Coefficient.

TABLE 4: The estimated parameters for the log-logistic AFT model.

\begin{tabular}{lcccc}
\hline \multirow{2}{*}{ Variable } & \multicolumn{4}{c}{ Log-logistic AFT model } \\
& Coef & SE & $z$-statistic & $p$ value \\
\hline (Intercept) & 1.635 & 0.076 & 21.570 & $<2 e-16$ \\
Response time & 0.072 & 0.002 & 39.970 & $<2 e-16$ \\
Traffic control & 0.220 & 0.030 & 7.470 & 0.000 \\
Disabled vehicle & 0.131 & 0.066 & 1.990 & 0.046 \\
Debris & -0.282 & 0.073 & -3.830 & 0.000 \\
Abandoned vehicle & -0.293 & 0.074 & -3.970 & 0.000 \\
Collision & 0.417 & 0.071 & 5.850 & 0.000 \\
Single lane blocked & 0.239 & 0.033 & 7.310 & 0.000 \\
Multiple lane blocked & 0.467 & 0.059 & 7.930 & 0.000 \\
All travel lanes blocked & 0.615 & 0.232 & 2.650 & 0.008 \\
Total closure & 0.633 & 0.176 & 3.600 & 0.000 \\
Injury involved & 0.418 & 0.072 & 5.830 & 0.000 \\
Fire involved & 0.731 & 0.200 & 3.650 & 0.000 \\
Night & -0.310 & 0.071 & -4.380 & 0.000 \\
Summer & -0.055 & 0.026 & -2.080 & 0.038 \\
\hline
\end{tabular}

clearance time. However, $p$ values generally overstate the evidence for an effect [46].

Turning to the model results, Table 5 shows the posterior effect probabilities and $p$ values of several variables. For a few variables (i.e., response time, traffic control, collision, multiple lane blocked, total closure, injury involved, and summer), the $p$ values from Cox $\mathrm{PH}$ model and the posterior effect probability $P(\beta \neq 0 \mid D)$ from the BMA model all indicate that there is a highly significant effect $(p<0.05$ and $P(\beta \neq 0 \mid D)=100 \%)$ on the clearance time. However, the BMA model and Cox PH model show the different conclusions for the variables in Table 5. The $p$ values from the Cox PH model overstate the evidence for the effects. For night, debris, and work zone involved, they are considered highly significant in terms of $p$ values $(p<0.01)$, while the posterior effect probabilities from the BMA model suggest that the evidence for an effect is not strong. Additionally, disabled vehicle, abandoned vehicle, and all travel lanes blocked are less significant determinant of incident clearance

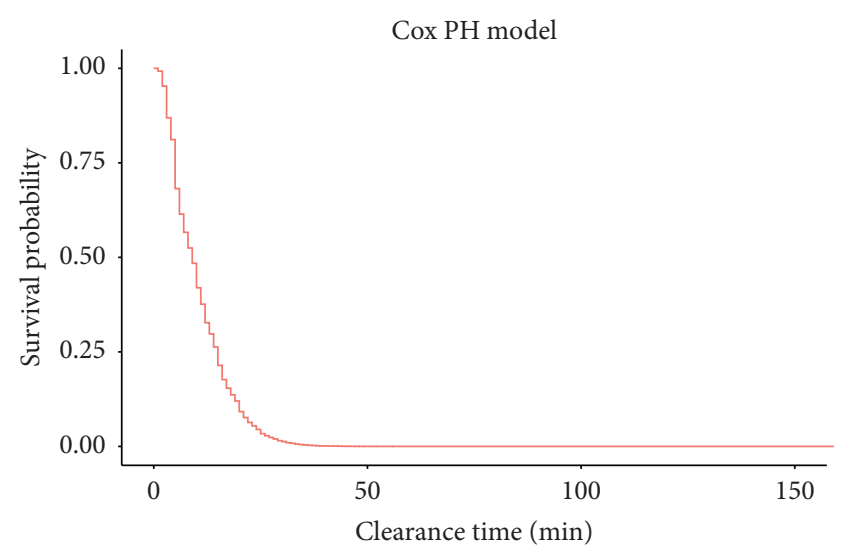

Figure 2: Estimated survival probability for the Cox PH model.

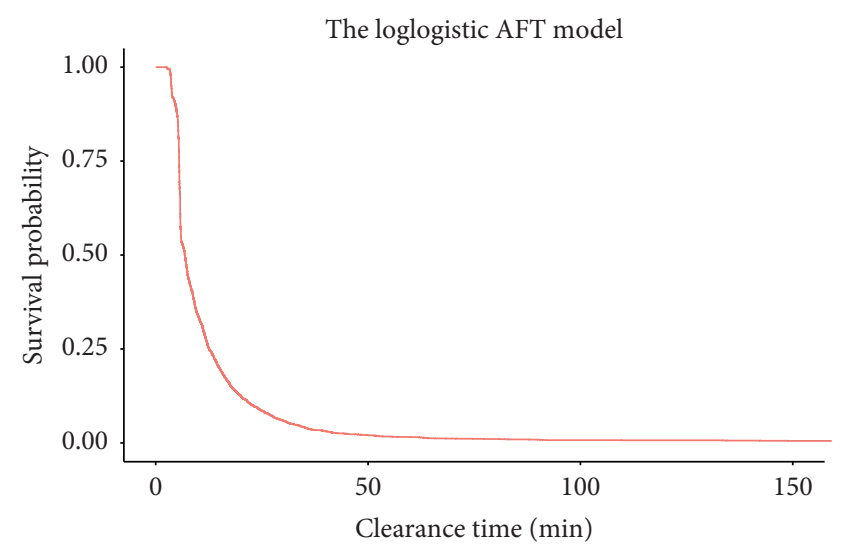

FIGURE 3: Estimated survival probability for the log-logistic AFT model.

time for $p$ values $(p<0.1)$, but the posterior effect probabilities provide weak evidence for the effect on incident clearance time. And for the last variable incident on HOV lane, both approaches show extremely low certainty evidence for an effect. Thus, the Cox PH model overstates the evidence for the effect on explanatory variables compared with the posterior effect probabilities $(P(\beta \neq 0 \mid D)<78 \%)$ for the BMA model according to the results in Table 5.

Furthermore, when rejecting the null hypothesis of "no effect," $p$ values used by the Cox PH model cannot distinguish between two scenarios, which are as follows: (a) there are few data to detect the effect on explanatory variables, and (b) the data provide evidence for the null hypothesis. However, the posterior effect probability in BMA can overcome the overstatement of the evidence for an effect resulting from the above issues. For instance, all travel lanes blocked and incidents on HOV lane are found to be a less significant determinant of incident clearance time for $p$ values and posterior effect probabilities. And for all travel lanes blocked, $P(\beta \neq 0 \mid D)$ equals $8.2 \%$, which is exactly described in scenario $(\mathrm{a})$, whereas the $P(\beta \neq 0 \mid D)=1.2 \%$ of incident on HOV lane indicates the evidence for the null hypothesis of "no effect." The latter can be approximated as the posterior probability with the effect small enough, 
TABLE 5: A comparison of some posterior effect probabilities from BMA and $p$ values from the Cox PH model.

\begin{tabular}{lcc}
\hline Variable & $\begin{array}{r}P(\beta \neq 0 \mid D)(\%) \\
(\mathrm{BMA})\end{array}$ & $\begin{array}{c}p \text { value }(\mathrm{Cox} \\
\mathrm{PH})\end{array}$ \\
\hline Night & 74.3 & 0.000 \\
Debris & 44.6 & 0.000 \\
Work zone involved & 15 & $3.9 e-07$ \\
Disabled vehicle & 77.7 & 0.019 \\
Abandoned vehicle & 23.7 & 0.034 \\
All travel lanes & 8.2 & 0.059 \\
blocked & 1.2 & 0.106 \\
Incident on Hov lane & & \\
\hline
\end{tabular}

namely, $P(|\beta|<\varepsilon)$, when $\varepsilon$ is less than half of a standard error [47].

3.1. Comparison of the Prediction Performance. As mentioned before, BMA can improve the prediction performance than any other single candidate model due to the overlook of model uncertainty for single selected models. The incident duration data were also applied to analyze the importance of each explanatory variable contributing to response variables by using two traditional survival analysis models. It is noted that the predicted values of Cox $\mathrm{PH}$ model are relative to the sample observations. Thus, we applied the nonparametric step-function (NPSF) approach to predict expected durations [48]. To further demonstrate the prediction performance of BMA, the Mean Absolute Error (MAE), the Root Mean Square Error (RMSE), and the mean absolute percentage error (MAPE) were used to measure the discrepancy between BMA and Cox PH model on assessing the accuracy. These performance indexes were calculated as $\mathrm{MAE}=(1 / n) \sum_{i=1}^{n}\left|O_{i}-P_{i}\right|, \quad \mathrm{RMSE}=(1$ /n) $\sqrt{\sum_{i=1}^{n}\left(O_{i}-P_{i}\right)^{2}}$ and MAPE $=(1 / n) \sum_{i=1}^{n}\left|\left(O_{i}-P_{i}\right) / O_{i}\right|$ $\times 100 \%$, where $O_{i}$ and $P_{i}$ are the observed value and the predicted value of clearance time for incident $i$, respectively. Smaller MAE, RMSE, and MAPE values indicate a better prediction performance.

In this study, 2,584 incident records were randomly divided into two subsets, one of which was used as training subdataset for the application of BMA and benchmark models, and the other is used as testing subdataset to measure prediction performance. The number of sections used to build and test the model was 1500 and 1084, respectively. To make the results more credible, training and testing subdataset varied according to ten random seeds selected.

Table 6 reports the mean of the prediction performance indexes from the BMA and Cox PH model, as well as the log-logistic AFT model. Bold values are the smallest MAE, RMSE, and MAPE values among three models. As shown in Table 6, multiple random experiments in this study indicate that the proposed BMA model results in better prediction performance in contrast with two traditional survival models in terms of three performance indexes. Thus, it can be concluded that the BMA model can improve the prediction performance for the conventional survival model.
TABle 6: Performance index values for the Cox PH, log-logistic AFT model and BMA models.

\begin{tabular}{lccc}
\hline Performance index & Cox PH & Log-logistic AFT model & BMA \\
\hline MAE & 6.051 & 6.325 & $\mathbf{5 . 9 6 8}$ \\
RMSE & 15.677 & 19.272 & $\mathbf{1 5 . 6 5 4}$ \\
MAPE & $48.791 \%$ & $48.728 \%$ & $\mathbf{4 8 . 3 2 0 \%}$ \\
\hline
\end{tabular}

\section{Conclusions}

This study has applied Bayesian Model Averaging (BMA) for incident clearance time analysis and prediction. The BMA approach was compared with the Cox Proportional Hazards model and Accelerated Failure Time model (with the loglogistic distribution) in analyzing the 2,584 freeway incident records obtained from I-5 corridor in Seattle. The main conclusions are summarized as follows: (1) Response time, traffic control, collision, multiple lane blocked, total closure, injury involved, and summer are the main factors affecting the duration of traffic incident clearance. (2) The modeling results from three considered models show discrepancy in estimated coefficients, such as response time, traffic control, and night. (3) Compared to the Cox PH and the log-logistic AFT model, the posterior effect probabilities for BMA can overcome the overstatement of the evidence for the effect on explanatory variables in contrast with $p$ values for a single model. (4) The prediction performance of BMA is better than the two classical survival models when predicting the traffic incident clearance time.

For future work, BMA can be applied to explain the effect of explanatory variables on incident clearance time or other phases of incident duration. Markov chain Monte Carlo model composition should be conducted to make a comparison with Occam's window method. Additionally, BMA can be extended to identify outliers in the clearance time data in terms of posterior model probabilities.

\section{Data Availability}

The data used to support the findings of this study are available from the corresponding author upon request.

\section{Conflicts of Interest}

The authors declare that they have no conflicts of interest.

\section{Acknowledgments}

This research was sponsored jointly by the National Key Research and Development Program of China (grant no. 2018YFE0102800), the Shanghai Science and Technology Committee (grant no. 19210745700) and the Fundamental Research Funds for the Central Universities (grant no. 22120210009).

\section{References}

[1] A. M. S. Alkaabi, D. Dissanayake, and R. Bird, “Analyzing clearance time of urban traffic accidents in Abu Dhabi, United Arab Emirates, with hazard-based duration modeling 
method," Transportation Research Record: Journal of the Transportation Research Board, vol. 2229, no. 1, pp. 46-54, 2011.

[2] H. Zhou and Z. Tian, "Modeling analysis of incident and roadway clearance time," Procedia-Social and Behavioral Sciences, vol. 43, pp. 349-355, 2012.

[3] Y. Zou, Y. Zhang, and K. Cheng, "Exploring the impact of climate and extreme weather on fatal traffic accidents," Sustainability, vol. 13, no. 1, p. 390, 2021.

[4] B. Ghosh, M. T. Asif, J. Dauwels, U. Fastenrath, and H. Guo, "Dynamic prediction of the incident duration using adaptive feature set," IEEE Transactions on Intelligent Transportation Systems, vol. 20, no. 11, pp. 4019-4031, 2019.

[5] D. Nam and F. Mannering, "An exploratory hazard-based analysis of highway incident duration," Transportation Research Part A: Policy and Practice, vol. 34, no. 2, pp. 85-102, 2000.

[6] R. Li, F. C. Pereira, and M. E. Ben-Akiva, "Overview of traffic incident duration analysis and prediction," European Transport Research Review, vol. 10, no. 2, 2018.

[7] Y. Wang, X. Ma, Z. Li, Y. Liu, M. Xu, and Y. Wang, "Profit distribution in collaborative multiple centers vehicle routing problem," Journal of Cleaner Production, vol. 144, pp. 203219, 2017.

[8] J. Tang, L. Zheng, C. Han et al., "Statistical and machinelearning methods for clearance time prediction of road incidents: a methodology review," Analytic Methods in Accident Research, vol. 27, Article ID 100123, 2020.

[9] X. Yang, Y. Zou, J. Tang, J. Liang, and M. Ijaz, "Evaluation of short-term freeway speed prediction based on periodic analysis using statistical models and machine learning models," Journal of Advanced Transportation, vol. 2020, Article ID 9628957, 16 pages, 2020.

[10] Y. Wang, S. Peng, X. Zhou, M. Mahmoudi, and L. Zhen, "Green logistics location-routing problem with eco-packages," Transportation Research Part E: Logistics and Transportation Review, vol. 143, Article ID 102118, 2020.

[11] Y. Wang, Y. Yuan, X. Guan et al., "Collaborative two-echelon multicenter vehicle routing optimization based on statespace-time network representation," Journal of Cleaner Production, vol. 258, Article ID 120590, 2020.

[12] A. Khattak, X. Wang, and H. Zhang, "Incident management integration tool: dynamically predicting incident durations, secondary incident occurrence and incident delays," IET Intelligent Transport Systems, vol. 6, no. 2, p. 204, 2012.

[13] J. Hong, R. Tamakloe, D. Park, and Y. Choi, "Estimating incident duration considering the unobserved heterogeneity of risk factors for trucks transporting HAZMAT on expressways," Transportation Research Record: Journal of the Transportation Research Board, vol. 2673, no. 2, pp. 232-242, 2019.

[14] R. Li, M. Guo, and H. Lu, "Analysis of the different duration stages of accidents with hazard-based model," International Journal of Intelligent Transportation Systems Research, vol. 15, no. 1, pp. 7-16, 2017.

[15] J.-T. Lee and J. Fazio, "Influential factors in freeway crash response and clearance times by emergency management services in peak periods," Traffic Injury Prevention, vol. 6, no. 4, pp. 331-339, 2005.

[16] R. Li and P. Shang, "Incident duration modeling using flexible parametric hazard-based models," Computational Intelligence and Neuroscience, vol. 2014, Article ID 723427, 10 pages, 2014.

[17] I. Ghosh, P. T. Savolainen, and T. J. Gates, "Examination of factors affecting freeway incident clearance times: a comparison of the generalized F model and several alternative nested models," Journal of Advanced Transportation, vol. 48, no. 6, pp. 471-485, 2014.

[18] H. J. Haule, T. Sando, R. Lentz, C.-H. Chuan, and P. Alluri, "Evaluating the impact and clearance duration of freeway incidents," International Journal of Transportation Science and Technology, vol. 8, no. 1, pp. 13-24, 2019.

[19] Y. Zou, K. Henrickson, D. Lord, Y. Wang, and K. Xu, "Application of finite mixture models for analysing freeway incident clearance time," Transportmetrica A: Transport Science, vol. 12, no. 2, pp. 99-115, 2016.

[20] A. J. Khattak, J. Liu, B. Wali, X. Li, and M. Ng, "Modeling traffic incident duration using quantile regression," Transportation Research Record: Journal of the Transportation Research Board, vol. 2554, no. 1, pp. 139-148, 2016.

[21] Y. Zou, J. Tang, L. Wu, K. Henrickson, and Y. Wang, "Quantile analysis of factors influencing the time taken to clear road traffic incidents," Proceedings of the Institution of Civil Engineers-Transport, vol. 170, pp. 1-9, 2017.

[22] Y. Zou, X. Ye, K. Henrickson, J. Tang, and Y. Wang, "Jointly analyzing freeway traffic incident clearance and response time using a copula-based approach," Transportation Research Part C: Emerging Technologies, vol. 86, pp. 171-182, 2018.

[23] H. Laman, S. Yasmin, and N. Eluru, "Joint modeling of traffic incident duration components (reporting, response, and clearance time): a copula-based approach," Transportation Research Record: Journal of the Transportation Research Board, vol. 2672, no. 30, pp. 76-89, 2018.

[24] Q. Shang, D. Tan, S. Gao, and L. Feng, "A hybrid method for traffic incident duration prediction using BOA-optimized random forest combined with neighborhood components analysis," Journal of Advanced Transportation, vol. 2019, Article ID 4202735, 11 pages, 2019.

[25] L. Li, X. Sheng, B. Du, Y. Wang, and B. Ran, “A deep fusion model based on restricted Boltzmann machines for traffic accident duration prediction," Engineering Applications of Artificial Intelligence, vol. 93, Article ID 103686, 2020.

[26] S.-b. Lee, D. H. Han, and Y.-I. Lee, "Development of freeway traffic incident clearance time prediction model by accident level," Journal of Korean Society of Transportation, vol. 33, no. 5, pp. 497-507, 2015.

[27] K. Ozbay and N. Noyan, "Estimation of incident clearance times using Bayesian networks approach," Accident Analysis \& Prevention, vol. 38, no. 3, pp. 542-555, 2006.

[28] S. Wang, R. Li, and M. Guo, "Application of nonparametric regression in predicting traffic incident duration," Transport, vol. 33, no. 1, pp. 22-31, 2015.

[29] J. Weng, L. Feng, G. Du, and H. Xiong, "Maximum likelihood regression tree with two-variable splitting scheme for subway incident delay," Transportmetrica A: Transport Science, vol. 15, no. 2, pp. 1061-1080, 2019.

[30] X. Ma, C. Ding, S. Luan, Y. Wang, and Y. Wang, "Prioritizing influential factors for freeway incident clearance time prediction using the gradient boosting decision trees method," IEEE Transactions on Intelligent Transportation Systems, vol. 18, no. 9, pp. 2303-2310, 2017.

[31] C. Zhan, A. Gan, and M. Hadi, "Prediction of lane clearance time of freeway incidents using the M5P tree algorithm," IEEE Transactions on Intelligent Transportation Systems, vol. 12, no. 4, pp. 1549-1557, 2011.

[32] J. Tang, L. Zheng, C. Han, F. Liu, and J. Cai, “Traffic incident clearance time prediction and influencing factor analysis using extreme gradient boosting model," Journal of Advanced Transportation, vol. 2020, Article ID 6401082, 12 pages, 2020. 
[33] A.-S. Mihaita, Z. Liu, C. Cai, and M.-A. Rizoiu, "Arterial incident duration prediction using a bi-level framework of extreme gradient-tree boosting," 2019, https://arxiv.org/abs/ 1905.12254 .

[34] Y. Zou, D. Lord, Y. Zhang, and Y. Peng, "Application of the bayesian model averaging in predicting motor vehicle crashes," in Proceedings of the Transportation Research Board 91st Annual Meeting, Washington, DC, USA, 2012.

[35] G. Blattenberger, R. Fowles, and P. D. Loeb, "Determinants of motor vehicle crash fatalities using Bayesian model selection methods," Research in Transportation Economics, vol. 43, no. 1, pp. 112-122, 2013.

[36] G. Li and J. Shi, "Application of Bayesian model averaging in modeling long-term wind speed distributions," Renewable Energy, vol. 35, no. 6, pp. 1192-1202, 2010.

[37] D. Draper, "Assessment and propagation of model uncertainty," Journal of the Royal Statistical Society: Series B (Methodological), vol. 57, no. 1, pp. 45-70, 1995.

[38] A. E. Raftery, D. Madigan, and J. A. Hoeting, "Bayesian model averaging for linear regression models," Journal of the American Statistical Association, vol. 92, no. 437, pp. 179-191, 1997.

[39] G. Kabir, S. Tesfamariam, and R. Sadiq, "Predicting water main failures using Bayesian model averaging and survival modelling approach," Reliability Engineering \& System Safety, vol. 142, pp. 498-514, 2015.

[40] J. Traczynski, "Firm default prediction: a Bayesian modelaveraging approach," Journal of Financial and Quantitative Analysis, vol. 52, no. 3, pp. 1211-1245, 2017.

[41] A. Tran, M. Pont, A. Aguirre, H. Durand, M. Crose, and P. D. Christofides, "Bayesian model averaging for estimating the spatial temperature distribution in a steam methane reforming furnace," Chemical Engineering Research and Design, vol. 131, pp. 465-487, 2018.

[42] D. Madigan and A. E. Raftery, "Model selection and accounting for model uncertainty in graphical models using Occam's window," Journal of the American Statistical Association, vol. 89, no. 428, pp. 1535-1546, 1994.

[43] A. E. Raftery, "Bayesian model selection in social research," Sociological Methodology, vol. 25, pp. 111-163, 1995.

[44] D. G. Kleinbaum and M. Klein, Survival Analysis, Springer, Berlin, Germany, 2010.

[45] L. Hou, Y. Lao, Y. Wang, Z. Zhang, Y. Zhang, and Z. Li, "Time-varying effects of influential factors on incident clearance time using a non-proportional hazard-based model," Transportation Research Part A: Policy and Practice, vol. 63, pp. 12-24, 2014.

[46] J. A. Hoeting, D. Madigan, A. E. Raftery, and C. T. Volinsky, "Bayesian model averaging: a tutorial," Statistical Science, vol. 14, no. 4, pp. 382-401, 1999.

[47] J. O. Berger and M. Delampady, "Testing precise hypotheses," Statistical Science, vol. 2, no. 3, pp. 317-335, 1987.

[48] J. Kropko and J. J. Harden, "Beyond the hazard ratio: generating expected durations from the cox proportional hazards model," British Journal of Political Science, vol. 50, no. 1, pp. 303-320, 2017. 